\title{
Recurrence in jejunal pouch after proximal gastrectomy for early upper gastric cancer
}

\author{
Masaki Nishimura $^{1}$, Ichiro Honda ${ }^{1}$, Satoshi Watanabe ${ }^{1}$, Matsuo Nagata ${ }^{1}$, Hiroaki Souda ${ }^{1}$, \\ and MASARU MiYAZAKI ${ }^{2}$ \\ ${ }^{1}$ Department of Gastroenterological Surgery, Chiba Cancer Center Hospital, Chiba, Japan \\ ${ }^{2}$ Department of General Surgery, Graduate School of Medicine, Chiba University, 1-8-1 Inohana, Chuo-ku, Chiba 260-8677, Japan
}

\begin{abstract}
Recently, jejunal pouch reconstruction after proximal gastrectomy has been a common procedure in Japan. However, according to our investigation, there are no reports of recurrence in the jejunal pouch. We experienced a patient with a recurrence in the jejunal pouch after proximal gastrectomy for early upper gastric cancer. In view of the pathological findings and the recurrence site, we suspected an implantation on the staple-line made for the jejunal pouch.
\end{abstract}

Key words Gastric cancer · Recurrence · Jejunal pouch interposition

\section{Introduction}

Recently, jejunal pouch reconstruction after proximal gastrectomy has been a common procedure in Japan. Remnant gastric cancer after proximal gastrectomy is rare as compared with distal gastrectomy, and recurrences in the remnant stomach following both types of gastrectomy are almost always in the stump, or they are recurrences of multiple cancers. There are no reports of recurrence in the jejunal pouch, according to our investigation. We report herein the case of a patient with jejunal pouch recurrence after proximal gastrectomy for early gastric cancer.

\section{Case report}

A 57-year-old woman with no history of gastric complaints had a stomach abnormality, found during a group test conducted in September 1997. At a local clinic, a circular elevated lesion in the anterior wall of the upper body of the stomach was discovered by upper

Offprint requests to: M. Miyazaki

Received: January 8, 2003 / Accepted: May 7, 2003 gastrointestinal endoscopy (Fig. 1). Gastrointestinal radiography showed type 1 gastric cancer (Fig. 2). A diagnosis of poorly differentiated adenocarcinoma was made by biopsy. The patient was referred to our hospital in October 1997 for treatment, and admitted.

The patient underwent proximal gastrectomy with jejunal pouch interposition (Fig. 3) on November 4, 1997. In the surgical procedure, the upper one-third of the stomach was resected using an automatic suturing device (the so-called Nakayama suturing instrument), and then a jejunal pouch was produced by means of another automatic suturing device (linear cutter). The tumor was diagnosed as stage A (med, sm1, INF $\beta$, ly1, v0, dm $32 \mathrm{~mm}, \mathrm{pm} 45 \mathrm{~mm}, \mathrm{n} 0$ [0/57], pN0, sH0, sP0, $\mathrm{cM} 0$ ), according to the general rules of the Japanese Gastric Cancer Association (JGCA). After the patient's discharge, we did not follow up with outpatient adjuvant therapy, because histological examination revealed that the cancer was limited to the submucosal layer, with no lymphatic metastases.

On March 27, 2000, a type 1 tumor located in the jejunal pouch was revealed through follow-up gastrointestinal endoscopy (Fig. 4). The biopsy specimen of this tumor indicated poorly differentiated adenocarcinoma. We surmised that this tumor was a recurrence, in the jejunal pouch, of the earlier gastric cancer. The patient was readmitted to our hospital for further examination and treatment. Results of physical examinations were unremarkable. Hematological studies revealed mild anemia (hemoglobin, $9.0 \mathrm{~g} / \mathrm{dl}$; hematocrit, $28 \%$ ). Results of other blood tests were within normal limits. The serum levels of alpha-fetoprotein (AFP), cancinoembryonic artigen (CEA), and carbohydrate antigen (CA)19-9 were within normal limits. On her first admission, tumor marker levels had been normal. We reviewed the examination findings on the first admission. Endoscopic findings included an elevated lesion (type 1) in the anterior wall of the gastric upper body (Fig. 1). Gastrointestinal radiography showed type 


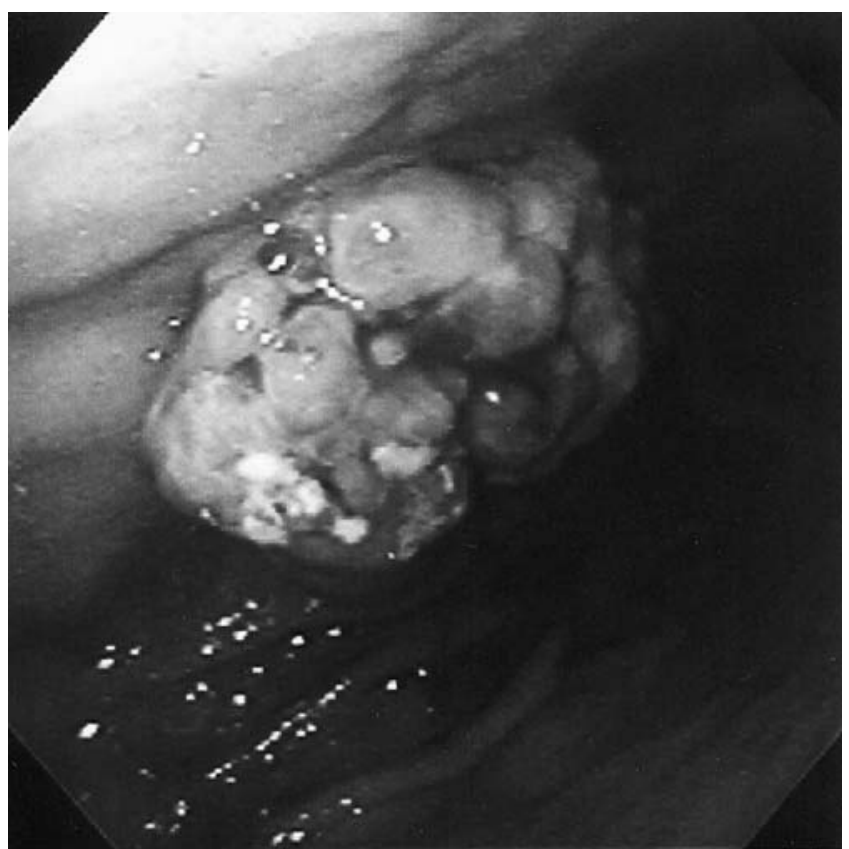

Fig. 1. Gastrointestinal endoscopy. The endoscopic finding was an elevated lesion (type 1) in the upper body of the stomach, diagnosed as poorly differentiated adenocarcinoma (por 1)

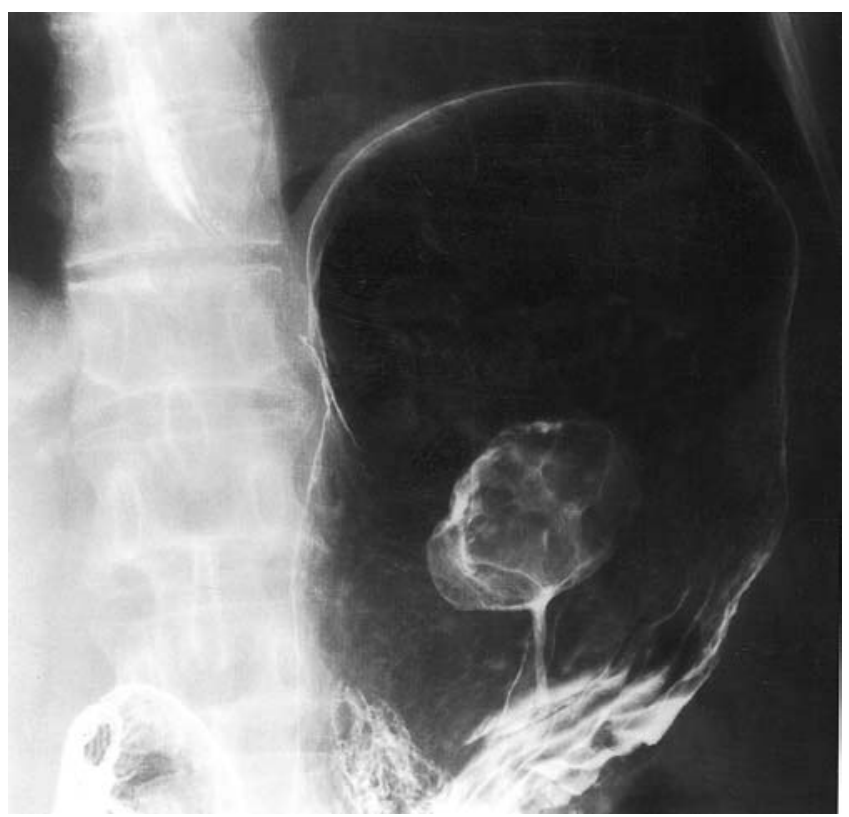

Fig. 2. Gastrointestinal radiography. Radiological examination shows type 1 gastric cancer in the upper body of the stomach

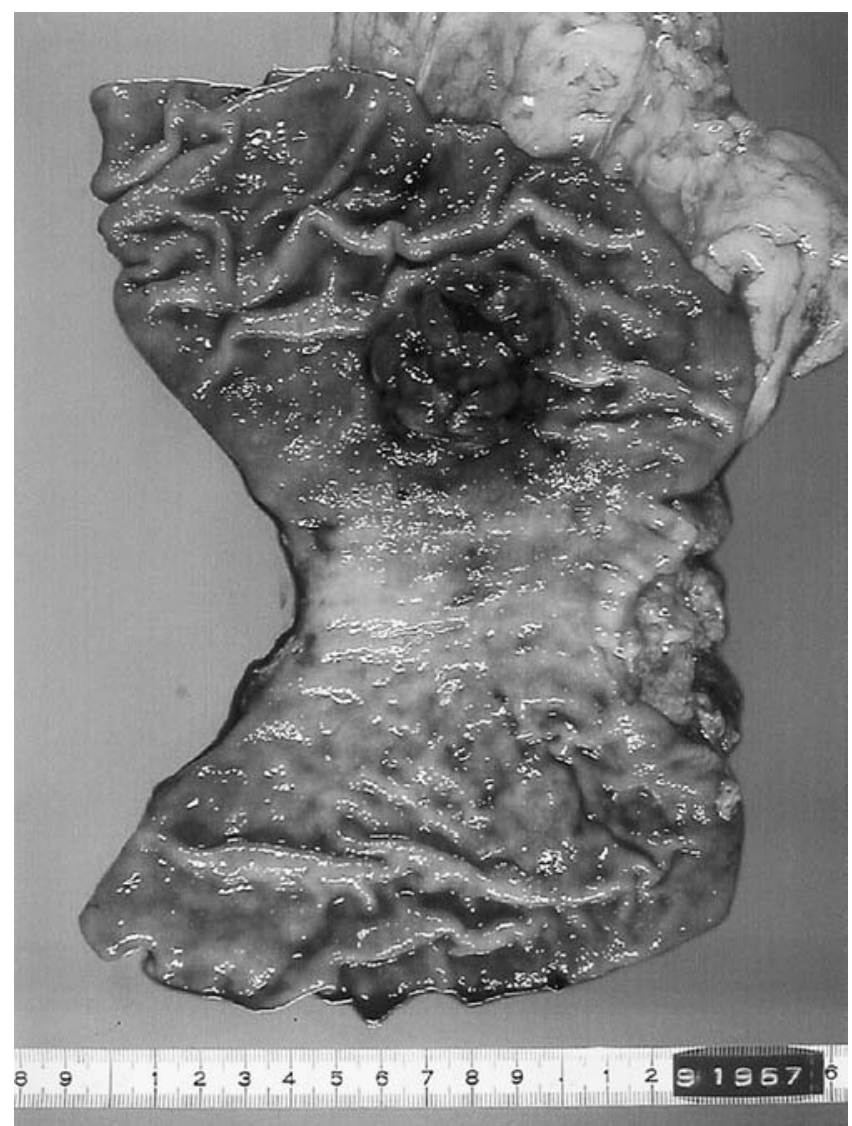

Fig. 3. Proximal gastrectomy specimen. Proximal gastrectomy was performed with jejunal pouch interposition $+\mathrm{D} 1$. The diagnosis was type 1 tumor, sSS, sN0, sP0, sH0, cM0; stage $\mathrm{B}$

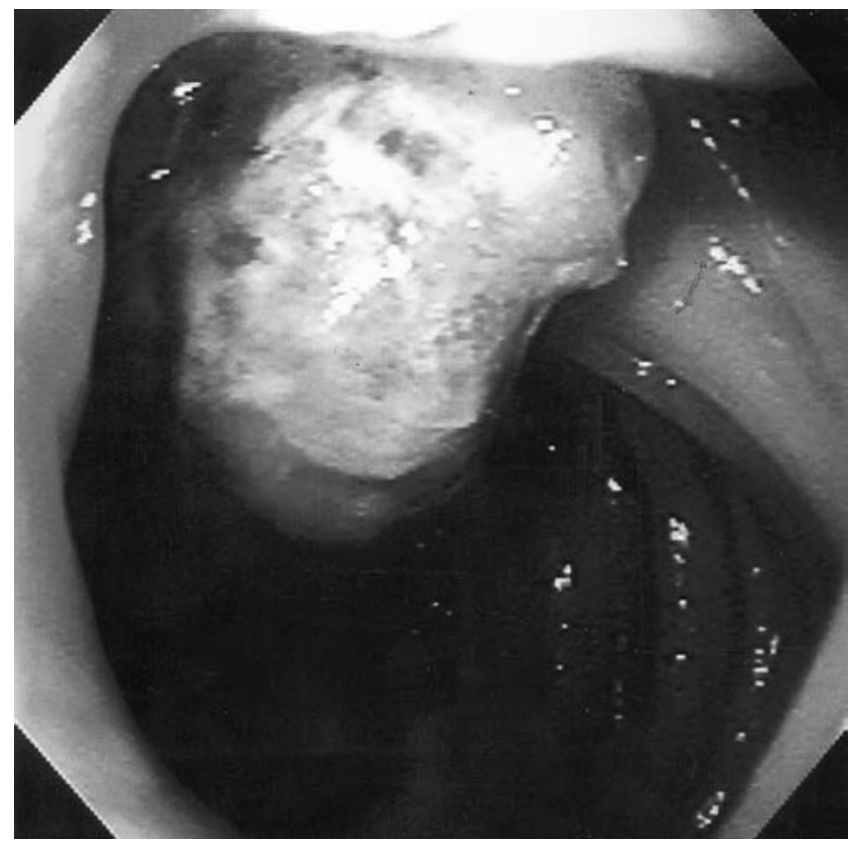

Fig. 4. Gastrointestinal endoscopy. An irregular elevated tumor grew in the jejunal pouch, and the tumor invaded the esophagus superior to the anastomosis 
1 gastric cancer (Fig. 2). A diagnosis of poorly differentiated adenocarcinoma was made by biopsy.

On examination at her second admission, gastrointestinal endoscopy revealed that an irregular, elevated tumor growing in the jejunal pouch had invaded the esophagus superior to the anastomosis (Fig. 4). On gastrointestinal radiography, the main tumor was located in the jejunal pouch, and involved the esophagus (Fig. 5). The tumor did not spread to the remnant stomach. A diagnosis of recurrence in the jejunal pouch after proximal gastrectomy for early upper gastric cancer was made, and we carried out a resection of the residual

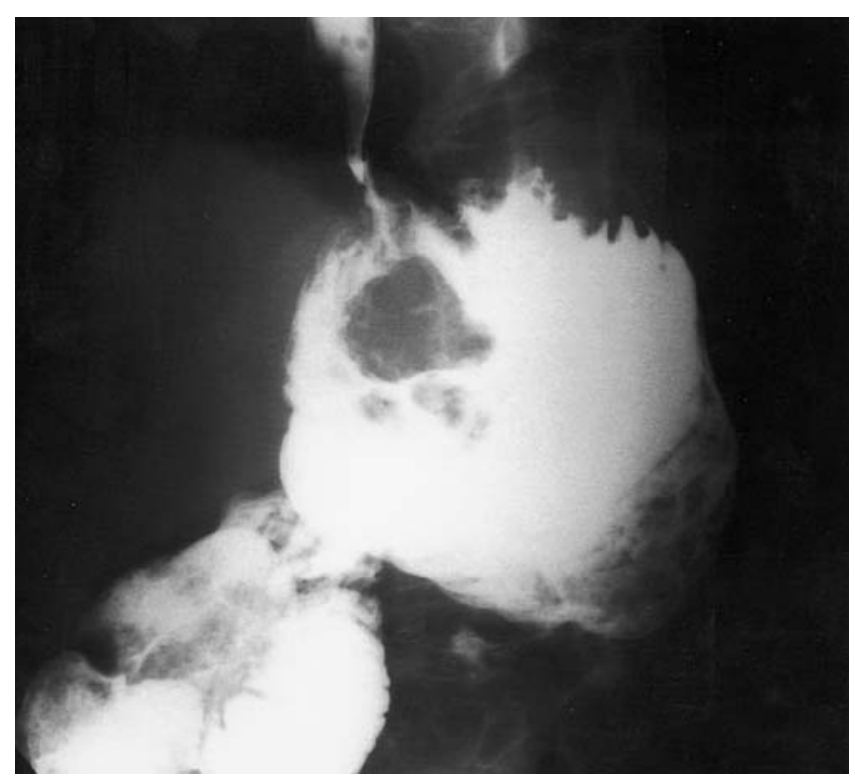

Fig. 5. Gastrointestinal radiography. The main tumor was located in the jejunal pouch and involved the esophagus; the tumor had not spread to the remnant stomach stomach and jejunal pouch, with Roux-en-Y reconstruction, in April 2000. The secondary tumor was classified as type 1 (Fig. 6). The histological diagnosis was poorly differentiated adenocarcinoma (por 1), med, se, INF $\beta$, ly $3, \mathrm{v} 2, \mathrm{pm} 8 \mathrm{~mm}, \mathrm{dm} 22 \mathrm{~cm}, \mathrm{n}(+)$ [3/28]. The esophagus was involved, but the remnant stomach was not involved. Pathological findings in regard to surgical specimens were similar to those regarding specimens from the first operation.

\section{Discussion}

Recurrence of gastric cancer in the remnant stomach after gastrectomy usually depends on stump recurrence, and other recurrences result from multiple cancers. Reports of recurrence of gastric cancer in the remnant stomach following proximal gastrectomy are rare as compared with distal gastrectomy. Only the report by Hiratsuka and Inagawa [1] noted as high a rate as 17 cases of stump recurrence after proximal gastrectomy among 191 cases of upper-portion gastric cancer, and they did not mention the reconstruction procedure. They pointed out four types of stump recurrence:

1. Remainder of stump (fDM $(+)$ )

2. Recurrence with lymphatic spread (submucosal or subserosal)

3. Implantation

4. Peritoneal dissemination

In our patient, the primary tumor was type 1 , from a macroscopic perspective. The tumor was $4.0 \mathrm{~cm}$ in diameter, and very soft and fragile, so that cancer cells detached easily from the primary cancer at the time of surgery. Histologically, it was poorly differentiated adenocarcinoma-medullary (por 1). The distal margin

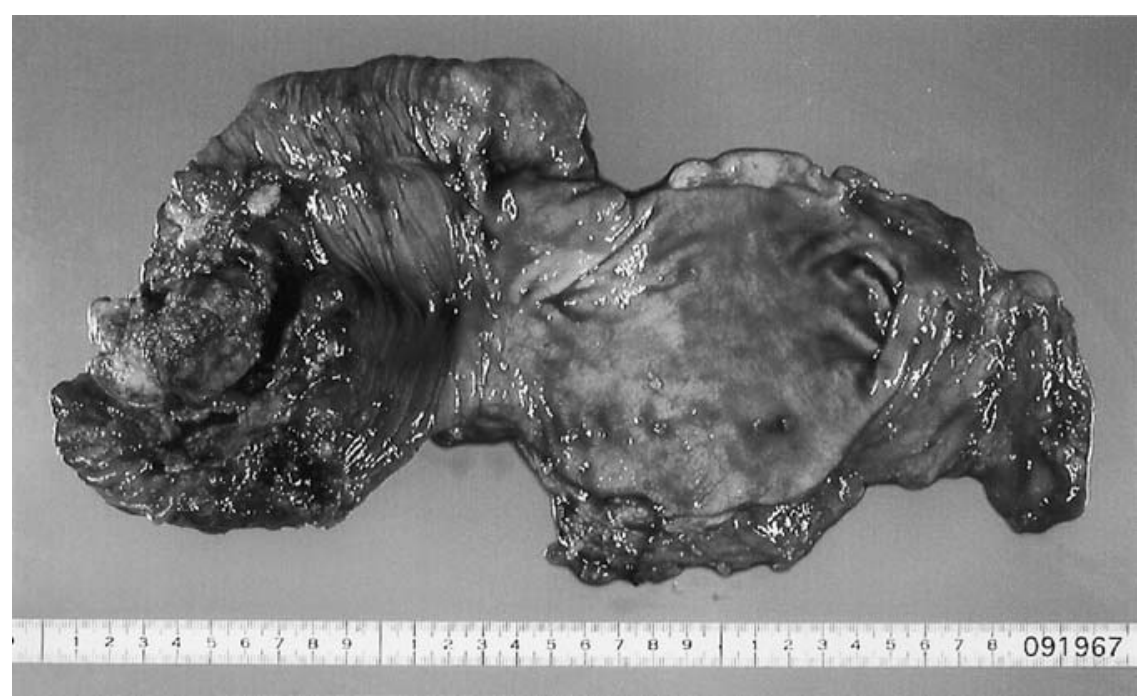

Fig. 6. Resected specimen from the second operation, which consisted of resection of the remnant stomach and jejunal pouch plus Roux-en-Y reconstruction 
was negative (pDM $(-)$ ), and the depth of invasion was $\mathrm{pSM}$. Lymphatic vessel invasion was positive (ly1), but there was no venous invasion (v0) and no lymph node involvement (pN0). The final stage was f1A (pT1pN0sH0sP0cM0CY0). At the time of the second operation, the tumor was located mainly in the jejunal pouch, and had invaded the abdominal esophagus to a distance of $0.3 \mathrm{~cm}$ in macroscopic terms, but there was no invasion of the remnant stomach, and no cancer therein. Histologically, it was poorly differentiated adenocarcinoma-medullary-(por 1), the depth of invasion was $\mathrm{pSE}, \mathrm{ly} 3, \mathrm{v} 2, \mathrm{pN} 2(+)$, and the final stage was $\mathrm{fB}$. The abdominal esophagus was involved, but the remnant stomach was free of cancer from the tumor and the tumor was $7.0 \mathrm{~cm}$ distant from the jejunogastric suture line. We carried out additional pathological examinations regarding the site of recurrence, with the help of pathologists. As a result, it appeared that the site of recurrence in this patient was the staple line of the jejunal pouch, in which the recurrent tumor was mainly located, with expansive growth from a macroscopic as well as a pathological perspective. It is therefore unlikely that cancer cells implanted in the esophagojejunal anastomosis proceeded into the jejunal pouch. Therefore, based on the report of Hiratsuka and Inagawa [1], two types of recurrence (in proximal gastrectomy, remainder of stump, and peritoneal dissemination) were rejected, and the two other possibilities were considered. One was recurrence with lymphatic spread (submucosal or subserosal); the other was implantation. However, we performed repeated additional pathological examinations of the noncancerous part, the stump of the esophagus as the proximal margin, and the gastric stump as the distal margin, and lymph vessel permeation (ly1) was found only inside the tumor, not in the noncancerous part or the oral margin. Therefore, we also judged that lymph vessel permeation was present in the ring obtained by automatic anastomosis, so there was little likelihood of lymphatic spread.

We find it quite possible that the stomach cancer was transplanted to the stapler line produced during the jejunal pouch reconstruction, via the linear stapler used in the first operation. In addition, it is possible that cancer cells that had attached to the nasogastric tube became detached after the operation, and then implanted on the stapler line. Similarly, Miyoshi et al. [2] reported a case of recurrence after a suture-line jejunal pouch procedure had been performed instead of total gastrectomy for advanced gastric cancer. In their patient, they proposed that the cause of the recurrence might have been implantation of exfoliated cancer cells in the jejunal mucosa used for the jejunal pouch, or contamination by cancer cells via the stapling devices. In our patient, however, the upper one-third of the stomach was resected by means of an automatic sutur- ing device (the so-called Nakayama suturing instrument), and then the jejunal pouch was formed by another automatic suturing device (linear cutter). Therefore, there was no possibility of contamination by cancer cells, via the instrument, on the staple line produced during the jejunal pouch reconstruction. Thus, we speculated that viable cancer cells had detached from the tumor removed in the remnant stomach, the esophagus, or from the jejunum used for the jejunal pouch, prior to its construction, and the detached cells then implanted on the staple line in the jejunal pouch. This case of recurrence is very similar to local recurrence in the large intestine and rectum. Surgical implantation from carcinoma of colon and rectum has long been known. In 1907 and 1908, Sir Charles Ryall [3,4] reported that loose, viable cancer cells may implant on freshly cut tissues during surgery for carcinoma of the large intestine and breast.

Umpleby [5] in a cytological examination, reported that exfoliated cells from colorectal cancer were viable, and that large numbers of cancer cells were shed into the intestinal lumen, implanting on the freshly reconstructed anastomosis. Slanetz [6] reported on the effects of "no-touch" isolation, which were achieved by controlling the lymphatic and luminal dissemination of malignant cells. In addition to minimizing tumor manipulation, bowel ligatures help to reduce cancerrelated deaths and the incidence of distant, local, and suture-line recurrence. Keynes [7] reported that mercury bichloride successfully reduced the incidence of free-cell implantation from the lumen of the large bowel, suggesting that some such irrigating agent should also be used more widely in cancer surgery involving organs other than the large intestine. Cohn [8] has reported the use of an iodized suture to control local implantation in the anastomotic site, and the use of a closed anastomosis, such as the "no-touch" isolation technique, to control tumor implantation. It is difficult to use the "no-touch" isolation technique in gastric surgery, but, if possible, it is quite appropriate to use irrigation and to disinfect the suture line, as is done for colorectal cancer, to prevent recurrence due to implantation that occurs at the time of operation.

Of note, the histological type of the primary tumor in our patient was por 1 (medullary type), which has a strong potential for metastasis and proliferation, and is very soft and fragile, so that cancer cells detach easily from the tumor during surgery. In surgical procedures for gastric cancer, surgical instruments such as automatic anastomosis devices and automatic suturing devices are used frequently. We suggest that it is important to avoid contamination through surgical instruments, in order to avoid staple-line recurrence, and that surgeons should carefully protect the cancerous organ. 


\section{References}

1. Hiratsuka M, Inagawa T. Recurrence in remnant stomach after gastrectomy; carcinoma of the remnant stomach. Iyaku Journal; 1995:112-22.

2. Miyoshi K, Fuchimoto S, Ohsaki T, Sakata T, Takeda K, Ohkawa $\mathrm{T}$, et al. Suture line recurrence in jejunal pouch replaced after total gastrectomy for gastric cancer. Gastric Cancer 1999;2:194-7.

3. Ryall C. Cancer infection and cancer recurrence. Lancet 1907;II: 1311-16.

4. Ryall C. The technique of cancer operations, with relation to the danger of cancer infection. BMJ 1908;2:1005-8.
5. Umpleby HC, Fermor B, Symes MO, Williamson RCN. Isolation of viable exfoliated colorectal cancer cells at the site of intestinal transection. Br J Surg 1983;70:680-97.

6. Slanetz CA Jr. Effect of no touch isolation on survival and recurrence in curative resections for colorectal cancer. Ann Surg Oncol 1998;5:390-8.

7. Keynes WM. Implantation from the bowel lumen in cancer of the large intestine. Ann Surg 1961;153:357-64.

8. Cohn I Jr. Implantation in cancer of the colon. Surg Gynecol Obstet 1967;501-8. 